\title{
Inhibition of histone deacetylase promotes a neuroprotective mechanism in an experimental model of Parkinson's disease
}

\author{
Hang $\mathrm{Li}^{1}$, Guolin Shi ${ }^{2}$, Hao Zha ${ }^{3}$, Liqing Zheng ${ }^{4}$, Zhan Luo ${ }^{5}$, Ying Wang ${ }^{6}$
}

\author{
${ }^{1}$ Department of Geriatrics, Chengdu Eighth People's Hospital (Geriatric Hospital \\ of Chengdu Medical College), Chengdu, Sichuan, China \\ ${ }^{2}$ Department of Neurosurgery, Second Affiliated Hospital of Kunming Medical \\ University, Kunming, Yunnan, China \\ ${ }^{3}$ Department of Reproductive and Genetics, Second Affiliated Hospital of Kunming \\ Medical University, Kunming, Yunnan, China \\ ${ }^{4}$ Talent Services Section, Chengdu Talent Service Centre for Healthcare Professionals, \\ Chengdu, Sichuan, China \\ ${ }^{5}$ Department of Physical Examination, Chengdu First People's Hospital, Chengdu, \\ Sichuan, China \\ ${ }^{6}$ Department of Neurology, Second Affiliated Hospital of Kunming Medical University, \\ Kunming, Yunnan, China
}

Submitted: 18 September 2020

Accepted: 7 November 2020

Arch Med Sci

DOI: https://doi.org/10.5114/aoms/130287

Copyright (c 2021 Termedia \& Banach

\begin{abstract}
A bstract
Introduction: Therapies targeting histone deacetylase (HDAC) have gained wider attention in the treatment of various clinical conditions. However, the use of HDAC inhibitors in pre-clinical trials in the case of Parkinson's disease (PD) is very limited. In the present study, the HDAC inhibitor, entinostat, was tested in animals induced with Parkinson's disease experimentally.

Material and methods: Wistar male rats $(150 \pm 10 \mathrm{~g})$ were administered with rotenone $(2 \mathrm{mg} / \mathrm{kg} /$ day, s.c.) for 21 days to induce PD, while entinostat $(20 \mathrm{mg} / \mathrm{kg}$ ) was given intraperitoneally. Then, the neurological functions, PD markers, and HDACs were analysed in the control and experimental animals. Results: The results demonstrated that rats that received entinostat displayed progressive motor, behavioural, and neurological function with attenuated $\alpha$-synuclein and improved tyrosine-hydroxylase compared to control cells. Moreover, the induction of PD in rats demonstrated reduced levels of $\mathrm{H} 2 \mathrm{~S}$, dopamine, 3, and 4-dihydroxyphenylacetic acid (DOPAC), and increased monoamine oxidase activity in PD rats. However, the rats that received entinostat demonstrated progressive levels of dopa and DOPAC, with attenuated levels of HDAC- $2,-4$, and -6 mRNA in the PD rats compared to controls. On the other hand, elevated $(p<0.01)$ levels of PD marker genes such as GDF3 and NMDA2b were reduced, with a significant increase in neuroprotective genes such as VDAC3 and CBX5 in entinostat-supplemented rats.

Conclusions: The study results suggest that inhibition of HDAC systematically improves the neurological functions, and hence treatments, emphasizing that $\mathrm{HDACl}$, as the speculated mechanism, will be a promising mode of treatment in PD.
\end{abstract}

Key words: Parkinson's disease, hydrogen sulphide, histone deacetylase, histone acetyltransferase, cytokines

\author{
Corresponding author: \\ Dr. Ying Wang \\ Department of Neurology \\ Second Affiliated \\ Hospital of Kunming \\ Medical University \\ Kunming, Yunnan \\ China 650101. \\ Phone/fax: \\ +86-0871-65351281 \\ E-mail: yingwang326@gmail. \\ com
}




\section{Introduction}

Parkinson's disease (PD) is a progressive neurodegenerative disease that affects the elderly in most parts of the world. The incidence of PD is increasing in all parts of the world, irrespective of the population [1, 2]. In Europe, it is known to be affecting about $1 \%$ of people above 60 years of age $[3,4]$. The disease has many motor symptoms such as bradykinesia, rigidity, and postural instability, and non-motor symptoms such as cognitive problems and disturbances in sleep patterns, and total body dysfunction [5]. There is a reduction in dopamine production in the substantia nigra compacta, and its delivery to the dorsal striatum is attributed to the decline in the patients' movement with PD [6]. This and the accumulation of the alpha-synuclein aggregate protein in the neurons [7] are PD's principal causes.

Healthy cells have a balanced state of action observed between histone acetyltransferases (HATs) and histone deacetylases (HDACs) in the acetylation and deacetylation of histone proteins and thereby the control of gene transcription $[8,9]$. The control of gene expression helps neurons maintain their homeostasis [8]. An imbalance in the activities of HATs and HDACs would result in a pathogenic state in which the balance is tilted towards histone deacetylation, which was observed in another neurodegenerative disease - Alzheimer's disease. In PD, $\alpha$-synuclein accumulation promotes histone PD hypoacetylation, as ascertained from both in vitro and in vivo research models [10]. In order to correct the tilt in the balance between HATs and HDACs, HDAC inhibitors are used to reduce cell death in the nigrostriatal pathways in $\operatorname{PD}[9,11,12]$.

Entinostat is a synthetic benzamide derivative that belongs to the substituted pyridyl carbamate class of HDAC-inhibiting compounds that can be used orally against HDACs I and IV classes [13, 14]. It promotes histone hyperacetylation and helps in the transcriptional activation of specific genes [15] that are involved in cell proliferation, differentiation, and apoptosis. These refer to the HDAC inhibiting activities of entinostat in the control of the solid tumour, and we are interested in testing this synthetic compound in the treatment of PD.

\section{Aim}

We propose herein that entinostat would act against PD by inhibiting the activity of HDAC and restoring the cellular functions and alleviating the influence of PD biomarkers in the pathogenesis of the disease.

\section{Material and methods}

\section{PD rat model}

For the study, Wistar male rats $(150 \pm 10 \mathrm{~g})$ were used. The experimental protocol was followed as per the Institutional Animal Ethical Committee's approval, and all animal experiments were carried out strictly in accordance with the institutional guidelines and suggestions from the Ethics Committee. All animals were kept in cages in a temperature-controlled room with a 12-hour light/dark cycle with temperature and humidity maintained at $25 \pm 2^{\circ} \mathrm{C}$ and $55 \pm 5 \%$, respectively. Rats were allowed free access to regular standard rat chow and water. A total of 48 rats were randomly allocated to 4 groups ( $n=12$ each): Group 1 as a sham control group; group 2 as a PD group (rotenone dissolved in $1 \%$ DMSO at the back of the neck) $[14,15]$; group 3 were given entinostat $(20 \mathrm{mg} / \mathrm{kg})$ intraperitoneally before the PD induction; and group 4 rats were given entinostat alone as drug control. The rats were maintained for 3 months. At the end of the experimental period, the animals were assessed for behavioural and neurological functions such as an elevated plus maze, forced swim test, Morris water maze, rotarod, and open-field tests, as described previously [16-18].

\section{Estimation of hydrogen sulphide, dopamine, DOPAC, and marker enzymes}

The estimation of hydrogen sulphide concentration in brain tissue and plasma samples was carried out as per the previous publications $[19,20]$ with few modifications per our tissue samples. This spectrophotometric method involves the reaction of sulphide with N,N-Dimethyl-p-phenylenediamine sulphate in the oxidizing agent $\mathrm{Fe}^{3+}$ in hydrochloric acid forming methylene blue that is read at $670 \mathrm{~nm}$. Furthermore, brain tissue extract sample was analysed for dopamine (DA) and DOPAC (3,4-dihydroxyphenylacetic acid) by high-performance liquid chromatography (HPLC) fitted with a Shimadzu L-ECD-6A electrochemical detector coupled with a Shim-Pak CLC- ODS column $(25 \mathrm{~cm}$ length), with flow volume of $0.5 \mathrm{ml} / \mathrm{min}$ and mobile phase comprised of $150 \mathrm{mM}$ monohydrate citric acid, $67 \mathrm{mM}$ sodium octyl sulphate, $2 \%$ tetrahydrofuran, and $4 \%$ acetonitrile in deionized water $(\mathrm{pH}$ 3.2). The analysts' retention time was determined using the standard monoamines, test samples were quantified by comparison with standards, and the results are expressed as ng/g wet tissue. Further monoamine oxidase (MAO) was assayed using commercial kits as per the manufacturer's instructions. Also, ELISA kits for $\alpha$-synuclein and tyrosine-hydroxylase were obtained from Fine Biotech, China, and commercial assay kits to analyse HDAC and HAT activity were obtained from Epigentek, USA.

\section{Reverse transcription-PCR}

In order to evaluate the expression of microRNA in the present study, quantitative RT-PCR was performed with miR-specific primers. RNA was isolat- 
ed from the brain tissues using Trizol reagent. For quantitative detection of miR-34b, miR-205, miR433, miR-19b, and miR-124, TaqMan MicroRNA reverse transcription kit, TaqMan miR specific assays, and snoRNA assays were used according to the manufacturer's instructions (Thermo Fisher Scientific, USA). SnoRNA was used as a control for normalization. To further elucidate the mRNA expression of PD markers, total RNA was extracted from the brain tissues using TRIzol ${ }^{\circledR}$ reagent. Briefly, brain tissues were homogenized in TRIzol and an equal amount of chloroform was added and centrifuged at 10,000 $\mathrm{g}$ for $15 \mathrm{~min}$ at $4^{\circ} \mathrm{C}$. The top aqueous phase was collected in a separate tube and mixed with isopropanol and incubated for $10 \mathrm{~min}$ at $25^{\circ} \mathrm{C}$ and later centrifuged at $12,000 \mathrm{~g}$ for $10 \mathrm{~min}$ at $4^{\circ} \mathrm{C}$. The obtained RNA pellet was washed with $70 \%$ ethanol, and the total RNA was quantified using a spectrophotometer. A known amount of RNA sample was transcribed to CDNA, and real-time PCR was done for specific genes using the $\mathrm{SYBR}^{\oplus}$ Green/ROX master mix (Qiagen). The gene-specific primers used in the study are listed in the Table I. The obtained $C t$ values were used to calculate gene expressions by the comparative Ct method ( $\triangle \Delta C T)$ using GAPDH as the housekeeping gene.

\section{Statistical analysis}

Statistical significance was evaluated with a one-way analysis of variance (ANOVA) and Stu- dent's $t$-test methods. Data were expressed as the mean \pm SEM. Graph Pad Prism version 5.0 was used for the analyses. Differences with a $p$-value of less than 0.05 were considered statistically significant.

\section{Results}

To evaluate the protective role of HDAC inhibition in alleviating Parkinson's disease (PD), the present study was conducted using the rat model of PD and administered with the HDAC inhibitor entinostat. Initially, the onset of PD was assessed using the neurological assessment, and the results demonstrated that significant reductions $(p<0.05)$ in the latency seen on the rotarod test apparatus were found in PD rats compared to the control group. In addition, the observed exploratory activity in the open field with anxious-like behaviour and depressive-like behaviour in PD-induced rats compared to controls. However, entinostat-administered rats restored the neurological functions in the PD rats (Figure 1).

Figure 2 shows the effect of entinostat on the levels of $\mathrm{H} 2 \mathrm{~S}$, and other metabolites of PD importance have been evaluated in control and PD-induced rats. The levels of $\mathrm{H}_{2} \mathrm{~S}$ were found to be decreased in both the plasma and brain tissues of PD-induced rats compared to control rats. Furthermore, the reduced levels of dopamine $(p<0.05), 3,4$-dihydroxyphenylacetic acid (DOPAC)

Table I. List of primer details

\begin{tabular}{|c|c|c|c|}
\hline Gene & Primer & Sequence & Annealing \\
\hline \multirow[t]{2}{*}{ Foxp3 } & $\mathrm{F}$ & AGAGTGCTAGAGCCAAGTGC & \multirow[t]{2}{*}{58} \\
\hline & $R$ & GGCCACTGTTAGGGTATGGG & \\
\hline \multirow[t]{2}{*}{ HDAC-1 } & $\mathrm{F}$ & ACTAGATAGGGACCAGCGCA & \multirow[t]{2}{*}{57} \\
\hline & $R$ & AGCTCCTAAGCAGGCACTTG & \\
\hline \multirow[t]{2}{*}{ HDAC-2 } & $\mathrm{F}$ & TCCGGAATGTTGCTCGATGT & \multirow[t]{2}{*}{59} \\
\hline & $R$ & TTGCATTTGAACACCAGGCG & \\
\hline \multirow[t]{2}{*}{ HDAC-3 } & $\mathrm{F}$ & ACACCCGATGAAACCCCATC & \multirow[t]{2}{*}{59} \\
\hline & $R$ & TCAGAATGGAAGCGGCACAT & \\
\hline \multirow[t]{2}{*}{ ATP5A1 } & $\mathrm{F}$ & AACAGCCTCTGCGTAACCAA & \multirow[t]{2}{*}{58} \\
\hline & $R$ & CCTCCCACCCAGGAAGTAGA & \\
\hline \multirow[t]{2}{*}{ VDAC3 } & $\mathrm{F}$ & CTACGGGCTCATCTTCACCC & \multirow[t]{2}{*}{57} \\
\hline & $R$ & GCCATAGATGGTCGGTCCAG & \\
\hline \multirow[t]{2}{*}{ CBX5 } & $\mathrm{F}$ & CGGTAGGTAAGGGAAGGCCA & \multirow[t]{2}{*}{58} \\
\hline & $R$ & GGGTATGTGTTGGTCCACCT & \\
\hline \multirow[t]{2}{*}{ GDF3 } & $\mathrm{F}$ & TGGTTCAGGATCGTGGTGTG & \multirow[t]{2}{*}{58} \\
\hline & $R$ & GGTGGCGGTTCCAATCCTTA & \\
\hline \multirow[t]{2}{*}{ GRIN2B } & $\mathrm{F}$ & GGGTCACGCAAAACCCTTTC & \multirow[t]{2}{*}{57} \\
\hline & $R$ & GGCTGACACCACTGGCTTAT & \\
\hline \multirow[t]{2}{*}{ GAPDH } & $\mathrm{F}$ & TACTGTTGTCCAGCTACGGC & \multirow[t]{2}{*}{59} \\
\hline & $R$ & CGACCAATGCGTCCAAATCC & \\
\hline
\end{tabular}


A

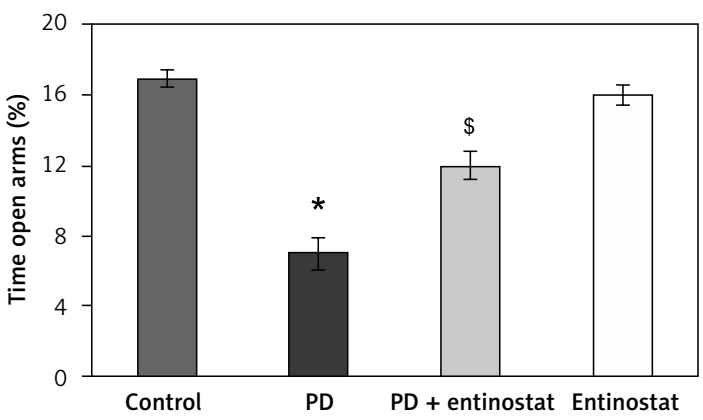

C

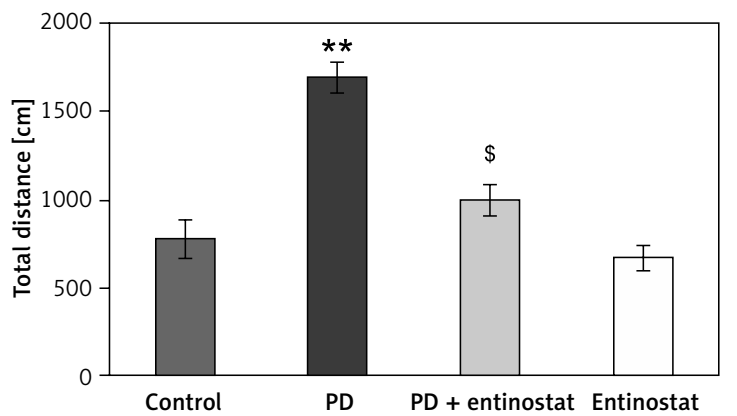

B

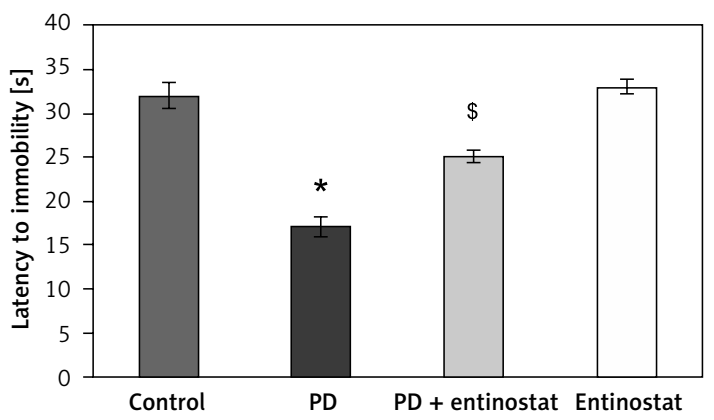

D

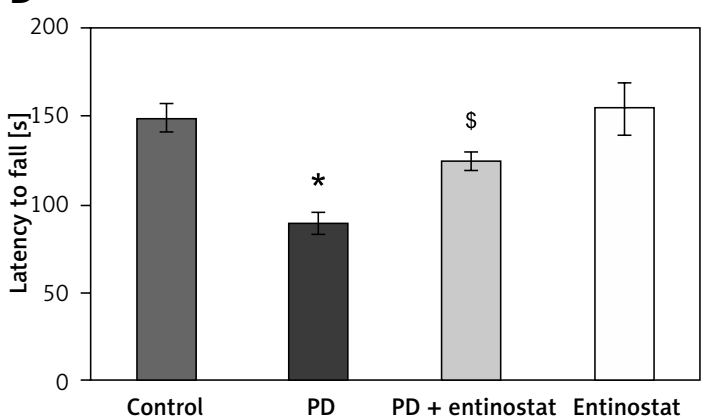

Figure 1. A-D - Represents the neurological function test such as elevated plus maze test, forced swim test, Morris water maze, and motor performance in the control and experimental groups of rats, respectively

Values are expressed as mean \pm S.E $(n=12)$. Statistical significance expressed as ${ }^{*} p<0.05,{ }^{* *} p<0.01$ compared to vehicletreated controls, ${ }^{s} p<0.05$ entinostat compared to $P D$ rats.

$(p<0.05)$, and tyrosine hydroxylase $(p<0.05)$ with increased levels of $\alpha$-synuclein and MAO activity and were observed in PD rats compared to controls. The administration of entinostat elevated the levels of $\mathrm{H}_{2} \mathrm{~S}(p<0.05)$ and restored the neurochemical equivalent to controls, suggesting protective effects of HDAC inhibition (Figure 2).

Moreover, to substantiate the role of HDAC inhibition on various HDAC genes classes, the mRNA levels of HDACs were identified by real-time quantitative PCR in relation to the control gene, and the results are shown in Figure 3. The results show a profound upsurge $(p<0.01)$ in the mRNA expression of Foxp 3 (3.2-fold), HDAC-1 (4.2-fold), HDAC-2 (2.6-fold), and HDAC-3 (3.4-fold) with increased HDAC activity and reduced HAT activity in PD rats compared to controls. However, the increased levels of these $H D A C$ genes were found to be reduced in entinostat treatment, indicating that the HDAC inhibition must be a major player in initiating the restorative mechanism in PD treatment (Figure 3).

Conversely, an additional experiment illuminating the PD marker gene levels was carried out, and the results are presented in Figure 4. In the present study, rats induced with PD using rotenone demonstrated a significant decrease $(p<$ $0.05)$ in the levels of mRNA such as ATP5A1 (-2.3fold), VDAC3 (-2.2-fold), and CBX5 (-3.3-fold) and increased levels of GDF3 (2.5-fold) and GRIN2B (2.7-fold) compared to controls. However, the lev- els of the genes that were restored in the group of PD rats receiving the HDAC inhibitor entinostat suggest its direct effect in cellular mechanisms involved in PD (Figure 4).

The role of HDAC signalling in PD was evaluated in terms of microRNA using qPCR. Figure 5 shows the expression level of miR-34b, miR-205, miR-433, miR-19b, and miR-124 in the control and experimental groups of rats. Rats induced with PD elicited a significant reduction $(p<0.05)$ in the expression of miR-34b (2.2-fold), miR-205 (1.7-fold), miR-433 (2.1-fold), miR-19b (2.3-fold), and miR-124 (2.6fold). On the other hand, the levels of these miRs were restored in entinostat pre-treatment, suggesting that the interplay of microRNAs is one of the clues in the aggravation of PD (Figure 5).

\section{Discussion}

In this study, we have discussed the putative role of HDAC in PD through the modulation of expression of various markers that are known to be associated with PD, and the potential role of entinostat as a new pharmacological molecule in the treatment of PD (Figure 6). $\mathrm{H}_{2} \mathrm{~S}$ is produced endogenously under physiological conditions for its role in the brain physiology. Patients suffering from neurodegenerative diseases like Alzheimer's [21] and PD [22] show decreased production of $\mathrm{H} 2 \mathrm{~S}$ in their brains. Our animal models showed that $\mathrm{H}_{2} \mathrm{~S}$ is decreased in the PD-induced group, and it 
A

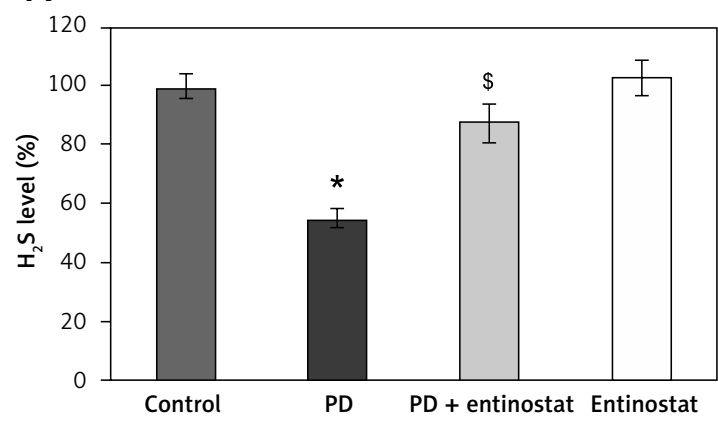

C

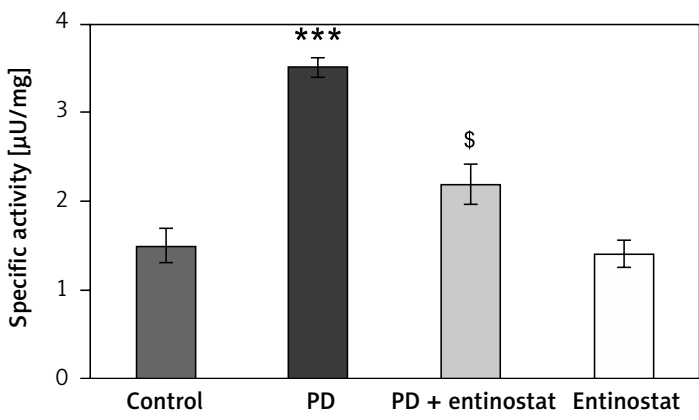

$\mathrm{E}$

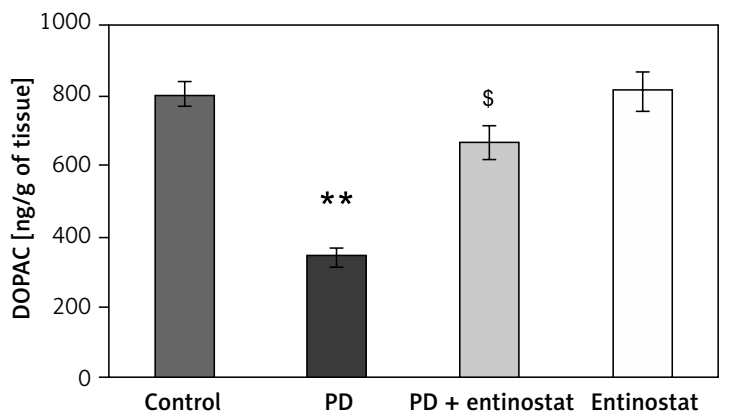

B

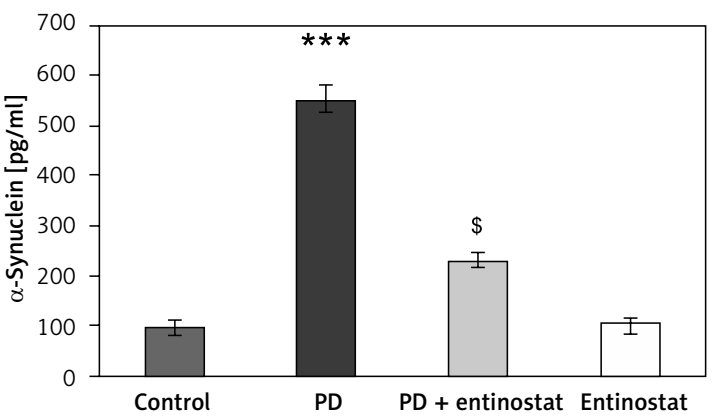

D

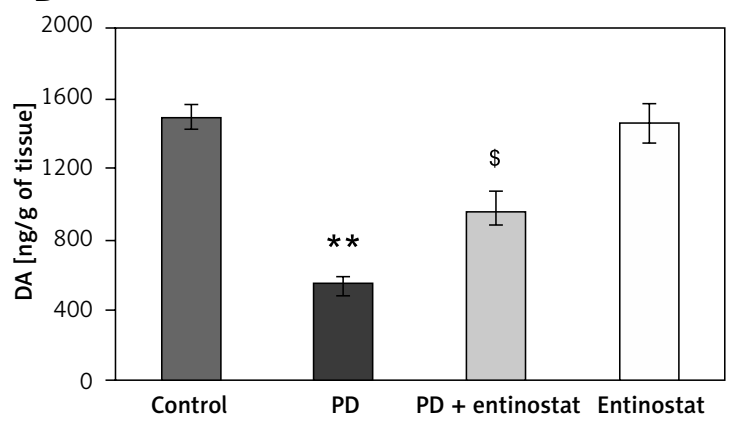

$F$

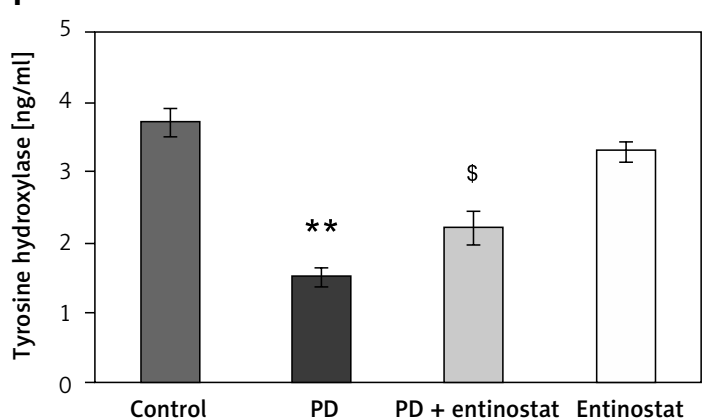

Figure 2. A-F - Represents the levels of $\mathrm{H}_{2} \mathrm{~S}$, DA, DOPAC, $\alpha$-synuclein, and marker enzymes of control and experimental groups of rats, respectively. The amount of DA and DOPAC is expressed as ng/g tissue. The amount of $\mathrm{H}_{2} \mathrm{~S}$ release was compared between groups and expressed as percentages

Values are expressed as mean $\pm S E(n=12)$. Statistical significance expressed as ${ }^{*} p<0.05,{ }^{* *} p<0.01$ compared to vehicletreated controls, ? $p<0.05$ entinostat compared to $P D$ rats.

correlates highly with the neurodegeneration [23]. Treatment with entinostat not only improved the $\mathrm{H}_{2} \mathrm{~S}$ production levels to near normalcy but also improved neuroprotection. With hydrogen sulphide providing neuroprotection to patients with Parkinson's disease [23], treatment with entinostat would have enhanced the hydrogen sulphide production and hence exerted its neuroprotective effects [24].

The level of expression of $\alpha$-synuclein is directly correlated with the PD phenotype, and the accumulation of $\alpha$-synuclein levels in the substantia nigra contributes to the PD pathogenesis [25]. In our models, the animals with the highest expression levels of $\alpha$-synuclein were induced with PD. The action of entinostat decreased the expression of $\alpha$-synuclein in the other successive groups. Similarly, the effect of PD is enhanced with increased activity of monoamine oxidase (MAO), which deaminates DA to generate a hydroxyl radical that contributes to the oxidative stress in PD [26]. MAO activity is increased in concordance with the increase in $\alpha$-synuclein to increase the oxidative stress in PD-induced rats, which is reduced in entinostat-treated groups. The action of entinostat follows its counterpart vorinostat in being a HDAC class I inhibitor $[9,27]$ in counteracting the effect of an increase of $\alpha$-synuclein by preventing its translocation to the nucleus for pathogenicity.

We assessed the ratios of levels of dopamine (DA): 3, 4-dihydroxyphenylacetic acid (DOPAC), which provides a biomarker of Parkinson's disease in patients with established synucleinopathies. The plasma and brain tissues of our PD-induced animals showed a decrease in the ratio of both DA and DOPAC, which is a typical early di- 
A

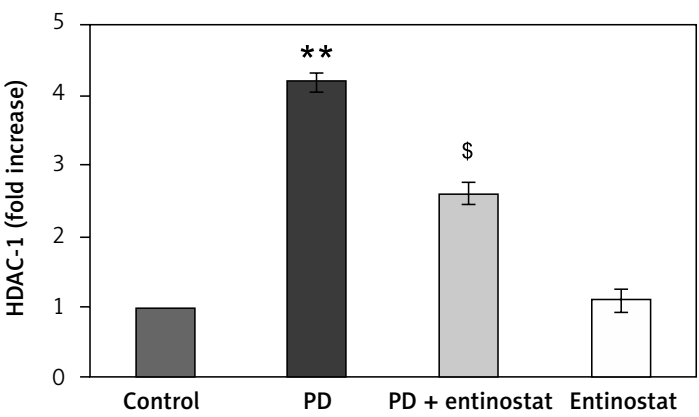

C

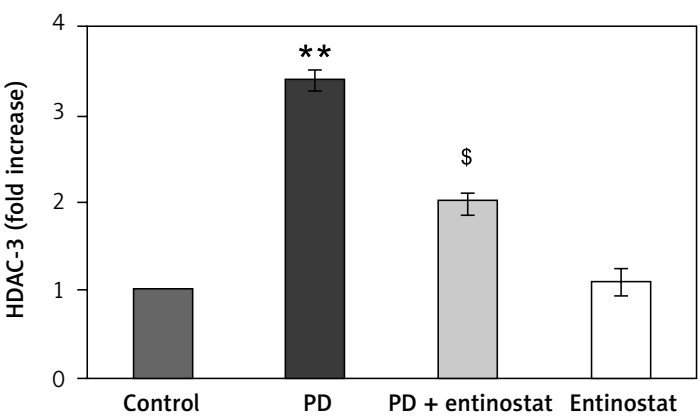

$\mathrm{E}$

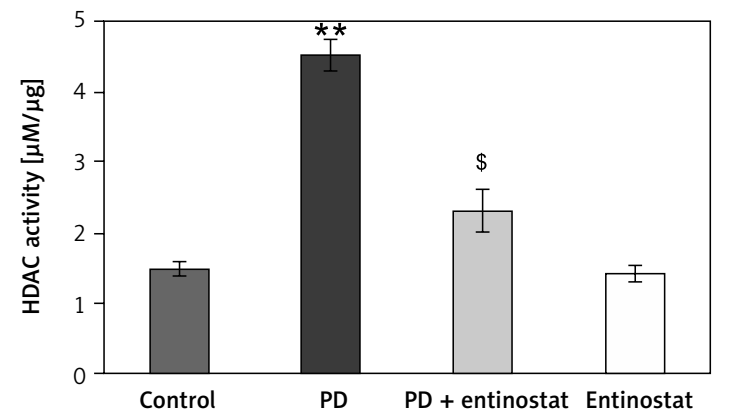

B

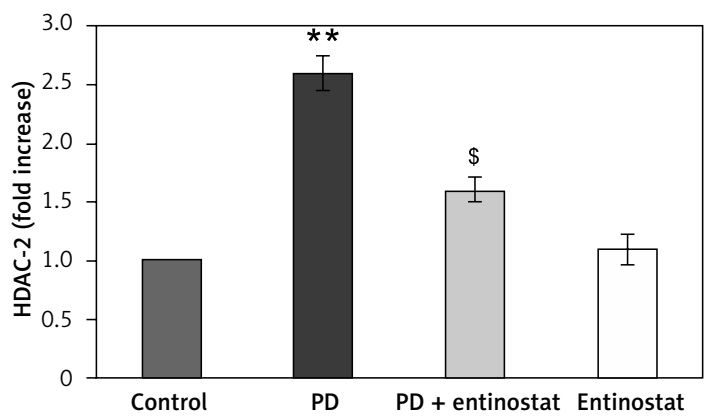

D

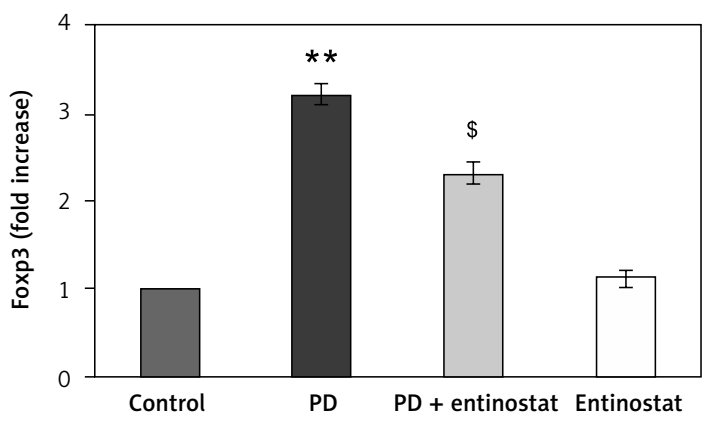

$\mathbf{F}$



Figure 3. A-F -Represents qRT-PCR mRNA expression analysis of HDAC genes in the control and experimental groups of rats. The fold increase of gene expression is compared with the housekeeping gene GAPDH. E, F - Total HDAC and HAT activity. The detail of the experiment is given in the methodology section

Values are expressed as mean $\pm S E(n=12)$. Statistical significance expressed as ${ }^{*} p<0.05,{ }^{* *} p<0.01$ compared to vehicletreated controls, ${ }^{s} p<0.05$ entinostat compared to $P D$ rats.

agnosis marker of Parkinson's. The depletion of DA from the nigrostriatum indicates PD, and the DOPAC levels in CSF are also decreased in patients with movement disorders [28]. These decreases in the PD animals were reversed in animals that were treated with entinostat, which increased the levels of DA and DOPAC.

A similar increase in tyrosine hydroxylase levels, which is a rate-limiting enzyme in the biosynthesis of DA [29], has been observed. Parkinson's disease is characterized by the loss of dopaminergic neurons and the loss of DA in the substantia nigra. The reduction in the levels of TH causes loss in DA synthesis, and this leads to PD. Thus, TH levels play a major role in the pathogenicity of PD. In cases where the synuclein increases, TH activity is compromised and decreases the DA synthesis, leading to PD [30]. The use of entinostat has effectively decreased the synuclein and increased the activity of $\mathrm{TH}$ to increase DA, and thereafter DOPAC synthesis, in the treated animals.

Histone deacetylases (HDACs) are enzymes that catalyse the removal of acetyl groups from lysine and arginine residues of amino-terminal tails of histones [31]. The removal of acetyl groups returned the chromatin to a condensed form that prevents the transcription of various genes from expression. The complete pattern of expression of HDACs would be ideal to find the targets for inhibiting them, and thus it could be used as a therapeutic strategy [12]. In order to find which of the HDAC isoforms are involved in the process of the pathophysiology of PD, we tested the mRNA levels of HDAC isoforms 1, 2, and 3 and found them 
A

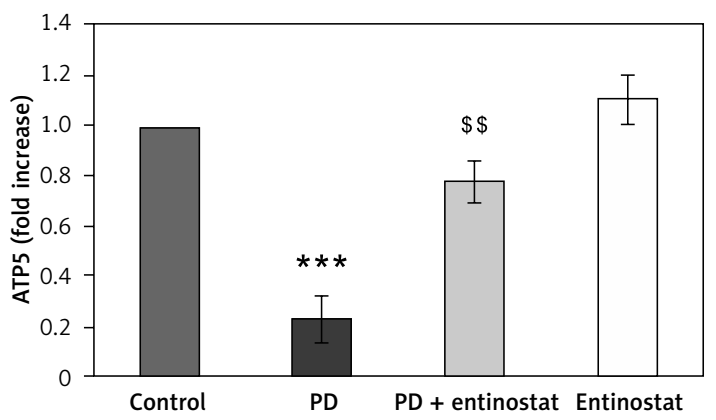

C

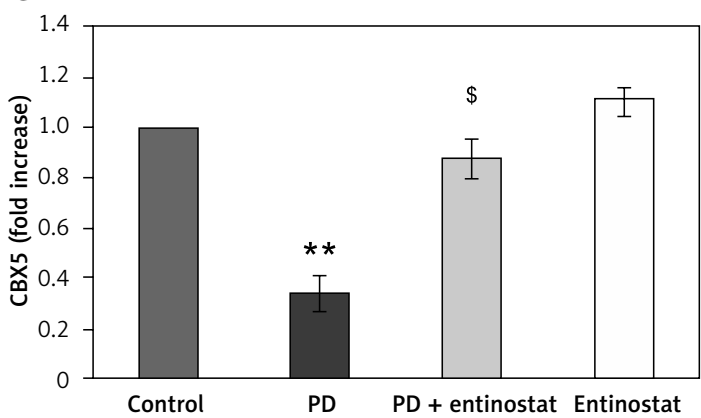

E

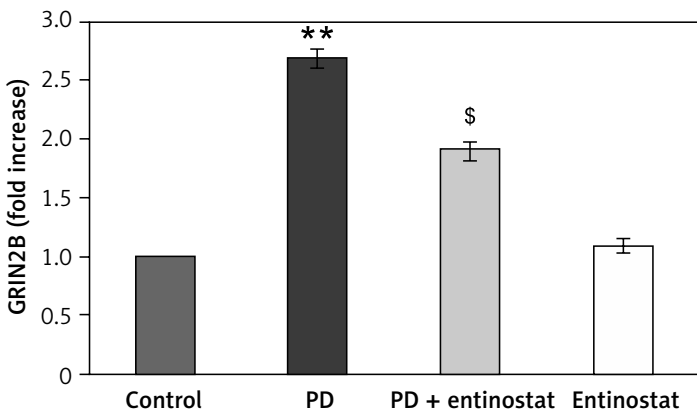

to be increased in all of the PD-induced animal groups, as already proven here [32], and hence a narrow-spectrum HDAC inhibitor is needed. When tested our molecular candidate for HDAC inhibition, entinostat significantly reduced the mRNA expression levels of HDACs 1, 2, and 3 in all the treated groups [33].

The HDAC inhibiting activity of entinostat was substantiated by a significant decrease in the HDAC activity, as researched here [34], and a simultaneous increase in the HAT activity was observed in the animals of the entinostat-treated group. We, for the first time, used entinostat in the HDAC inhibition in PD. Furthermore, entinostat suppresses Foxp 3 expression mRNA, leading to decreased Foxp3 protein, causing a defective impaired response on the Treg population to cause immunomodulation in PD [35]. These observations exhibited in our entinostat-treated group of animals are in line with established results, in which a similar decrease in the expression of Foxp 3 at the transcription level was observed $[35,36]$.
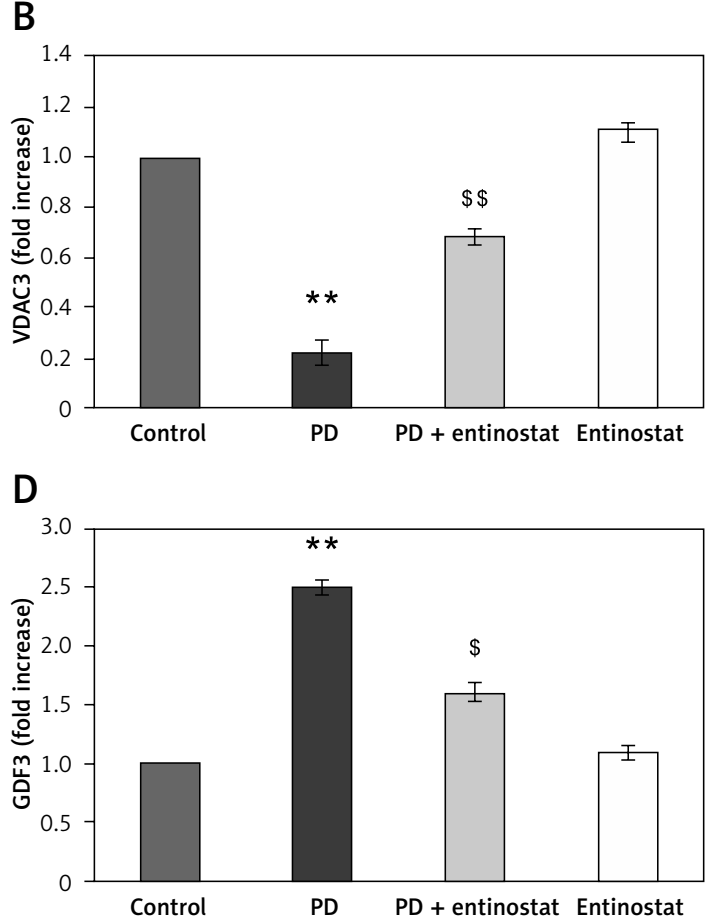

Figure 4. A-E - Represents qRT-PCR mRNA expression analysis of marker genes in the control and experimental groups of rats. The fold increase of gene expression is compared with the housekeeping gene GAPDH. The detail of the experiment is given in the methodology section

Values are expressed as mean $\pm S E(n=12)$. Statistical significance expressed as ${ }^{* *} p<0.01,{ }^{* * *} p<0.001$ compared to vehicle-treated controls, ${ }^{s} p<0.05,{ }^{s s} p<0.01$ entinostat compared to $P D$ rats.

Disruption of mitochondria and its metabolic activity are primarily focused on the pathogenesis of PD [37]. There is a dysfunction of mitochondria in neurodegenerative diseases utilizing very little glucose in the decreased ATP synthase activity with reduced energy production $[38,39]$. These are known to be the hallmarks of neurodegenerative diseases. Accompanying this would be the decrease in ATP5A1 subunits that oxidative impairment has destabilized the protein as well as its enzymatic activity [40]. The ATP5A1 expression is severely compromised in the PD-animal group because the data fits well with the established results of neurodegeneration and subsequent impairment of brain metabolism [39]. The expression and activity are restored with the action of entinostat in the treated group due to its mitochondria preservative activity and by maintaining ATP $[41,42]$. The pathologies of VDAC isoform 3 can be attributed to its altered levels of gene expression $[43,44]$. Conversely, the present study results also reflect decreased isoform 3 expression 
A

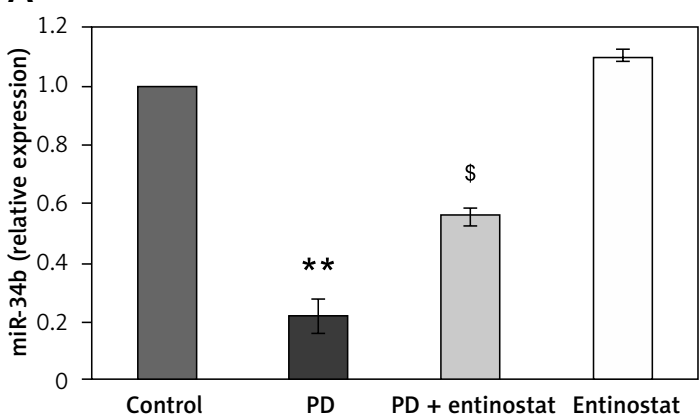

C


in the PD-animal group and returned to near normalcy in the entinostat-treated group.

Much of the gene expression profiling already performed to differentiate between the PD patients and healthy controls identifies ATP synthase subunit $\alpha$ (ATP5A1), voltage-dependent anion-selective channel protein 3 (VDAC-3) [45], growth differentiation factor 3 (GDF3) [46], glutamate receptors, glutamate ionotropic receptor NMDA type subunit 2B (GRIN2B) [47], chromobox homologue 5, and $C B X 5$, confirmed by $q R T-P C R$ to be found in the substantia nigra dopaminergic neuron degeneration [48] or acting upon mitochondria in the pathogenesis of PD. More studies are needed to elucidate the exact roles that they play in PD animals beyond the scope of PD marker identification.

Small non-coding RNAs, miRs, bind to the 3'UTR of the mRNA and regulate the gene expression, and dysregulation of miR expression is associated with PD and other neurodegenerative diseases $[49,50]$. Hence, we explored the miRs that are involved in PD pathogenesis. The impor-
B

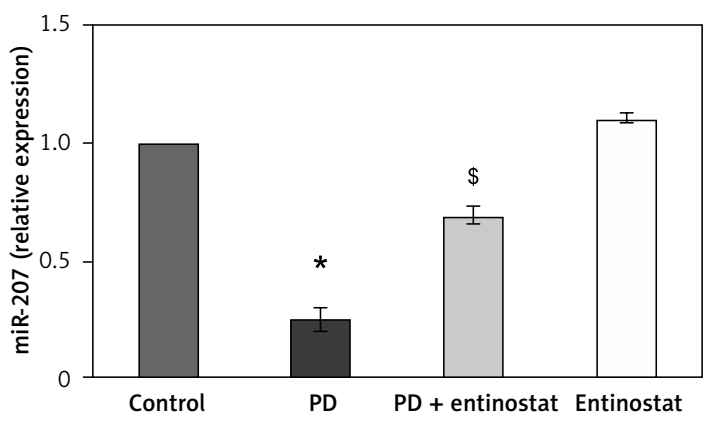

D

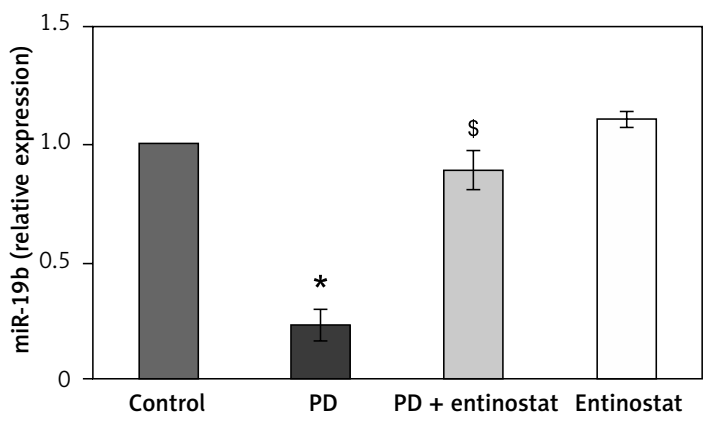

Figure 5. A-E - Represents expression analysis of microRNAs such as miR-34b, miR-205, miR-433, miR-19b, and miR-124 in the control and experimental groups of rats, respectively. The detail of the experiment is given in the methodology section Values are expressed as mean $\pm S E(n=12)$. Statistical significance expressed as ${ }^{*} p<0.05,{ }^{* *} p<0.01$ compared to vehicle-treated controls, ${ }^{s} p<0.05$ entinostat compared to $P D$ rats.

tance of miR-34b in PD pathogenesis is known due to the fact that it has low expression in parts of the brain in PD patients [51]. When the expression of miR-34b is reduced, oxidative stress increases with decreased mitochondrial metabolism and decreased viability of dopaminergic neuronal cells. Also, its inhibition can increase the $\alpha$-synuclein levels towards PD pathogenesis [52]. Treatment with entinostat restored the cell viability, mitochondrial function, and downregulation of ROS in the animals. The expression of miR-433 was found to be decreased in PD patients' serum and CSF and is in line [34] with our results in which a decrease in the expression of miR-433 is observed in PD-induced animals. The increase in alpha-synuclein or under conditions of synucleinopathies, miR-19b, was known to be downregulated and also serves as a biomarker for PD. These increases in pathogenic miR have been countered by the corresponding increase in miR-124, which is highly expressed in the brain and provides a neuroprotective role in PD, which is highly expressed in en- 


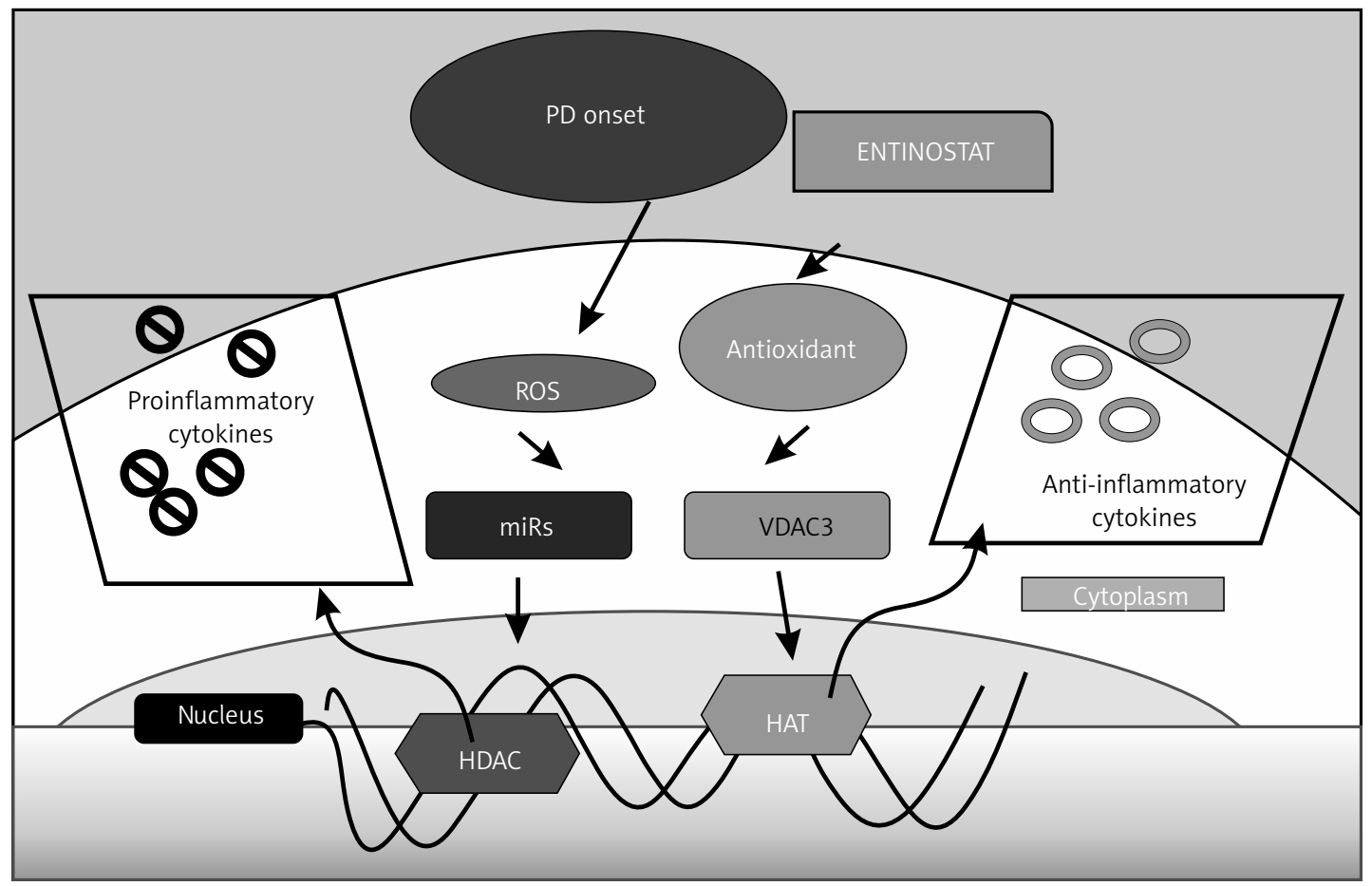

Figure 6. Schematic representation of the probable mechanisms by which entinostat exerts inhibition on cell dysfunction in PD. PD onset enhances ROS release activation with depletion of an antioxidant defence mechanism, which further activates the unregulated shuttling of proinflammatory cytokines with enhanced activation of HDAC with suppressed HAT activity. However, treatment with entinostat enhanced the antioxidant enzymes with regulated levels of deranged cytokines, and HDAC genes suggest that HDAC inhibition can ameliorate PD-mediated harmful cytokines

tinostat-treated animals (Figure 6). More studies are required to decode the mechanism by which entinostat reduces Parkinson's miR markers from their effect on the physiology of the disease. The results obtained are not specific because these markers are well known as biomarkers of PD.

In conclusion, our study results elucidate partial molecular mechanisms by which the PD animals relieve their neurodegenerative symptoms and restore their coordinated movements with the rehabilitation of mitochondria that are the primary site of pathogenesis in $\mathrm{PD}$, as well as by alleviating the biomarkers of PD by its primary action on HDAC. Entinostat would thus serve as a general and an epigenetic regulator against HDACs. Further mechanisms by which this molecule would act require a more systematic approach involving recent genetic findings and would be a better neuroprotective mediator of animal models with PD.

\section{Conflict of interest}

The authors declare no conflict of interest.

\section{References}

1. Pringsheim T, Jette N, Frolkis A, et al. The prevalence of Parkinson's disease: a systematic review and metaanalysis. Mov Disord 2014; 29: 1583-90.
2. Sun Y, Li D, Su Y, et al. Protective effect of hydrogen sulfide is mediated by negative regulation of epigenetic histone acetylation in Parkinson's disease. Arch Med Sci 2020. https://doi.org/10.5114/aoms.2020.93121.

3. De Lau LM, Breteler MM. Epidemiology of Parkinson's disease. Lancet Neurol 2006; 5: 525-35.

4. Wei L, He F, Zhang W, et al. Analysis of master transcription factors related to Parkinson's disease through the gene transcription regulatory network. Arch Med Sci 2019. doi:10.5114/aoms.2019.89460.

5. Jankovic J. Parkinson's disease: clinical features and diagnosis. J Neurol Neurosurg Psychiatry 2008; 79: 368-76.

6. Van Heesbeen HJ, Smidt MP. Entanglement of genetics and epigenetics in Parkinson's disease. Front Neurosci 2019; 13: 277.

7. Labbe C, Lorenzo-Betancor O, Ross OA. Epigenetic regulation in Parkinson's disease. Acta Neuropathol 2016; 132: 515-30.

8. Saha RN, Pahan K. HATs and HDACs in neurodegeneration: a tale of disconcerted acetylation homeostasis. Cell Death Differ 2006; 13: 539-50.

9. Dietz KC, Casaccia P. HDAC inhibitors and neurodegeneration: at the edge between protection and damage. Pharmacol Res 2010; 62: 11-7.

10. Kontopoulos E, Parvin JD, Feany MB. Alpha-synuclein acts in the nucleus to inhibit histone acetylation and promote neurotoxicity. Hum Mol Genet 2006; 15: 3012-23.

11. Kazantsev AG, Thompson LM. Therapeutic application of histone deacetylase inhibitors for central nervous system disorders. Nat Rev Drug Discov 2008; 7: 854-68.

12. Harrison IF, Dexter DT. Epigenetic targeting of histone deacetylase: therapeutic potential in Parkinson's disease? Pharmacol Ther 2013; 140: 34-52. 
13. Saito A, Yamashita T, Mariko Y, et al. A synthetic inhibitor of histone deacetylase, MS-27-275, with marked in vivo antitumor activity against human tumors. Proc Natl Acad Sci USA 1999; 96: 4592-7.

14. Suzuki T, Ando T, Tsuchiya K, et al. Synthesis and histone deacetylase inhibitory activity of new benzamide derivatives. J Med Chem 1999; 42: 3001-3.

15. Xu WS, Parmigiani RB, Marks PA. Histone deacetylase inhibitors: molecular mechanisms of action. Oncogene 2007; 26: 5541-52.

16. Campos FL, Carvalho MM, Cristovao AC, et al. Rodent models of Parkinson's disease: beyond the motor symptomatology. Front Behav Neurosci 2013; 7: 175.

17. Johnson ME, Bobrovskaya L. An update on the rotenone models of Parkinson's disease: their ability to reproduce the features of clinical disease and model gene-environment interactions. Neurotoxicology 2015; 46: 101-16.

18. Hausser N, Johnson K, Parsley MA, et al. Detecting behavioral deficits in rats after traumatic brain injury. J Vis Exp 2018; 131: 56044.

19. Zheng Y, Liao F, Du JB, et al. Modified methylene blue method for measurement of hydrogen sulfide level in plasma. Sheng Li Xue Bao 2012; 64: 681-6.

20. Dutta M, Biswas UK, Chakraborty R, et al. Evaluation of plasma H2S levels and $\mathrm{H} 2 \mathrm{~S}$ synthesis in streptozotocin-induced type-2 diabetes-an experimental study based on Swietenia macrophylla seeds. Asian Pac J Trop Biomed 2014; 4 (Suppl 1): S483-7.

21. Eto K, Asada T, Arima K, et al. Brain hydrogen sulfide is severely decreased in Alzheimer's disease. Biochem Biophys Res Commun 2002; 293: 1485-8.

22. Cao X, Cao L, Ding L, et al. A New hope for a devastating disease: hydrogen sulfide in Parkinson's disease. Mol Neurobiol 2018; 55: 3789-99.

23. Xue X, Bian JS. Neuroprotective effects of hydrogen sulfide in Parkinson's disease animal models: methods and protocols. Methods Enzymol 2015; 554: 169-86.

24. Cao P, Liang Y, Gao X, et al. Administration of MS-275 improves cognitive performance and reduces cell death following traumatic brain injury in rats. CNS Neurosci Ther 2013; 19: 337-45.

25. Stefanis L. alpha-Synuclein in Parkinson's disease. Cold Spring Harb Perspect Med 2012; 2: a009399.

26. Sandri G, Panfili E, Ernster L. Hydrogen peroxide production by monoamine oxidase in isolated rat-brain mitochondria: its effect on glutathione levels and $\mathrm{Ca} 2+$ efflux. Biochim Biophys Acta 1990; 1035: 300-5.

27. Goers J, Manning-Bog AB, McCormack AL, et al. Nuclear localization of alpha-synuclein and its interaction with histones. Biochemistry 2003; 42: 8465-71.

28. Goldstein DS, Holmes C, Sullivan P, et al. Elevated cerebrospinal fluid ratios of cysteinyl-dopamine/3,4-dihydroxyphenylacetic acid in parkinsonian synucleinopathies. Parkinsonism Relat Disord 2016; 31: 79-86.

29. Haavik J, Toska K. Tyrosine hydroxylase and Parkinson's disease. Mol Neurobiol 1998; 16: 285-309.

30. Zhu Y, Zhang J, Zeng Y. Overview of tyrosine hydroxylase in Parkinson's disease. CNS Neurol Disord Drug Targets 2012; 11: 350-8.

31. Seto E, Yoshida M. Erasers of histone acetylation: the histone deacetylase enzymes. Cold Spring Harb Perspect Biol 2014; 6: a018713.

32. Broide RS, Redwine JM, Aftahi N, et al. Distribution of histone deacetylases 1-11 in the rat brain. J Mol Neurosci 2007; 31: 47-58.

33. Zhu S, Denman CJ, Cobanoglu ZS, et al. The narrow-spectrum HDAC inhibitor entinostat enhances NKG2D ex- pression without NK cell toxicity, leading to enhanced recognition of cancer cells. Pharm Res 2015; 32: 779-92. 34. Zhang XR, Yang BL, Hu P, et al. Reduced circulating levels of miR-433 and miR-133b are potential biomarkers for Parkinson's disease. Front Cell Neurosci 2017; 11: 170.

35. Shen L, Pili R, Class. I histone deacetylase inhibition is a novel mechanism to target regulatory $T$ cells in immunotherapy. Oncoimmunology 2012; 1: 948-50.

36. Shen L, Ciesielski M, Ramakrishnan S, et al. Class I histone deacetylase inhibitor entinostat suppresses regulatory $\mathrm{T}$ cells and enhances immunotherapies in renal and prostate cancer models. PLoS One 2012; 7: e30815.

37. Barrera G, Gentile F, Pizzimenti S, et al. Mitochondrial dysfunction in cancer and neurodegenerative diseases: spotlight on fatty acid oxidation and lipoperoxidation products. Antioxidants 2016; $5: 7$.

38. Schagger H, Ohm TG, Human diseases with defects in oxidative phosphorylation. 2. F1F0 ATP-synthase defects in Alzheimer's disease revealed by blue native polyacrylamide gel electrophoresis. Eur J Biochem 1995; 227: 916-21.

39. Rhein V, Eckert A. Effects of Alzheimer's amyloid-beta and tau protein on mitochondrial function: role of glucose metabolism and insulin signalling. Arch Physiol Biochem 2007; 113: 131-41.

40. Perluigi M, Sultana R, Cenini G, et al. Redox proteomics identification of 4-hydroxynonenal-modified brain proteins in Alzheimer's disease: role of lipid peroxidation in Alzheimer's disease pathogenesis. Proteomics Clin Appl 2009; 3: 682-93.

41. Manavalan A, Mishra M, Feng L, et al. Brain site-specific proteome changes in ageing-related dementia. Exp Mol Med 2013; 45: e39.

42. Baltan S, Murphy SP, Danilov CA, et al. Histone deacetylase inhibitors preserve white matter structure and function during ischemia by conserving ATP and reducing excitotoxicity. J Neuro Sci 2011; 31: 3990-9.

43. Desruisseaux, MS, lacobas DA, lacobas S, et al. Alterations in the brain transcriptome in plasmodium berghei ANKA infected mice. J Neuroparasitology 2010; 1: n100803.

44. Chakravarty S, Reddy BR, Sudhakar SR, et al. Chronic unpredictable stress (CUS)-induced anxiety and related mood disorders in a zebrafish model: altered brain proteome profile implicates mitochondrial dysfunction. PLoS One 2013; 8: e63302.

45. Rocha S, Freitas A, Guimaraes SC, et al. Biological implications of differential expression of mitochondrialshaping proteins in Parkinson's disease. Antioxidants 2017; 7: 1.

46. Simunovic F, Yi M, Wang Y, et al. Gene expression profiling of substantia nigra dopamine neurons: further insights into Parkinson's disease pathology. Brain 2009; 132: 1795-809.

47. Michel PP, Alvarez-Fischer D, Guerreiro S, et al. Role of activity-dependent mechanisms in the control of dopaminergic neuron survival. J Neurochem 2007; 101: 289-97.

48. Calligaris R, Banica M, Roncaglia P, et al. Blood transcriptomics of drug-naive sporadic Parkinson's disease patients. BMC Genomics 2015; 16: 876.

49. Yelamanchili SV, Fox HS. Defining larger roles for "tiny" RNA molecules: role of miRNAs in neurodegeneration research. J Neuroimmune Pharmacol 2010; 5: 63-9.

50. Qiu L, Zhang W, Tan EK, et al. Deciphering the function and regulation of microRNAs in Alzheimer's disease and Parkinson's disease. ACS Chem Neurosci 2014; 5: 884-94. 
51. Minones-Moyano E, Porta S, Escaramis G, et al. MicroRNA profiling of Parkinson's disease brains identifies early downregulation of $\mathrm{miR}-34 \mathrm{~b} / \mathrm{c}$, which modulate mitochondrial function. Hum Mol Genet 2011; 20: 3067-78.

52. Kabaria S, Choi DC, Chaudhuri AD, et al. Inhibition of miR-34b and miR-34c enhances alpha-synuclein expression in Parkinson's disease. FEBS Lett 2015; 589: 319-25. 